\title{
ESTUDIO EXPLORATORIO DE LA PRESENCIA DE MICROCONTAMINANTES EN EL CICLO URBANO DEL AGUA EN COLOMBIA: CASO DE ESTUDIO SANTIAGO DE CALI
}

\author{
Carlos A. MADERA-PARRA ${ }^{1}$, Eliana M. JIMÉNEZ-BAMBAGUE ${ }^{1 *}$, Andrés F. TORO-VÉLEZ ${ }^{2}$, \\ Jaime A. LARA-BORRERO ${ }^{3}$, Diego F. BEDOYA-RÍOS ${ }^{3}$ y Verónica DUQUE-PARDO ${ }^{3}$
}

${ }^{1}$ Escuela de Ingeniería de Recursos Naturales y del Ambiente, Universidad del Valle. Calle 13, No. 100-00, Ciudad Universitaria Meléndez, Santiago de Cali, Colombia, C. P. 76001

${ }^{2}$ Grupo de Saneamiento Ambiental, Universidad del Valle. Calle 13, No. 100-00, Ciudad Universitaria Meléndez, Santiago de Cali, Colombia, C. P. 76001

${ }^{3}$ Grupo de Investigación Ciencia e Ingeniería del Agua y el Ambiente, Pontificia Universidad Javeriana. Carrera 7, No. 40-62, Bogotá D.C., Colombia, C. P. 110231

*Autor para correspondencia: jimenez.eliana@correounivalle.edu.co

(Recibido marzo 2017; aceptado octubre 2017)

Palabras clave: compuestos farmacéuticos, disruptores endocrinos, agua potable, agua residual, agua superficial

\section{RESUMEN}

Los microcontaminantes incluyen una amplia gama de compuestos presentes en el ambiente en concentraciones de $\mu \mathrm{g} / \mathrm{L}$ o ng/L. En este estudio se analizó la presencia y concentración de microcontaminantes como compuestos farmacéuticos y disruptores endocrinos en el ciclo urbano del agua de la ciudad de Santiago de Cali, Colombia, donde se incluyeron cuatro puntos de monitoreo de agua superficial, tres de agua potable y uno de agua residual. Se realizaron cinco jornadas de muestreo: un muestreo preliminar en el cual se cuantificaron 92 compuestos clasificados en cinco grupos de compuestos farmacéuticos (I, II, III, IV y V) y un grupo de disruptores endocrinos (DE), a partir del cual se priorizaron tres grupos a ser determinados en los cuatro muestreos siguientes. Los criterios de presencia, concentración, efectos sobre la biota acuática y financiamiento de la investigación, se emplearon para seleccionar los grupos I, IV y DE, los cuales tuvieron una presencia superior al $48 \%$ y una concentración media de hasta $11000 \mathrm{ng} / \mathrm{L}$. Se encontró que los compuestos más relevantes, en términos de presencia y concentración en el ciclo urbano del agua fueron 10,11-dihydro-10,11-dihidroxicarbamazepina, ibuprofeno, naproxeno, gemfibrozilo, paracetamol, sulfametoxazol, estronas, 4-iso-nonilfenol y bisfenol A. La presencia en el ciclo urbano del agua de Cali genera una alerta por la potencial amenaza sobre los organismos acuáticos y la salud humana.

Key words: pharmaceutical compounds, endocrine disruptors, drinking water, wastewater, superficial water

\begin{abstract}
Micropollutants includes a wide variety of compounds in environmental concentrations of $\mu \mathrm{g} / \mathrm{L}$ or $\mathrm{ng} / \mathrm{L}$. The presence and concentration of micropollutants, as pharmaceutical and endocrine disruptors compounds, were studied throughout the urban water cycle of Santiago de Cali city, Colombia. In order to do this, samples were collected in four points of surface water, three of drinking water and one of wastewater. Five samplings were carried out: one screening with 92 compounds measured, classified in five pharmaceutical compounds groups (I, II, III, IV and V) and one endocrine disruptors group
\end{abstract}


(ED), from which three groups were selected to be measured in the following four samplings. Under criteria of occurrence, concentration, environmental effects and financial support; the I, IV and ED groups were selected, with occurrence higher than $48 \%$ and mean concentration up to $11000 \mathrm{ng} / \mathrm{L}$. In terms of occurrence and concentration, the compounds 10,11-dihydro-10,11-dihydroxycarbamazepine, ibuprofen, naproxen, gemfibrozilo, paracetamol, sulfamethoxazole, estrone, 4-iso-nonylphenol and bisphenol A were relevant. The occurrence in urban water cycle of Cali provides a warning due to the potential threat to aquatic organism and human health.

\section{INTRODUCCIÓN}

Los microcontaminantes (MC) incluyen sustancias químicas como pesticidas, farmacéuticos, productos de cuidado personal, plastificantes, hidrocarburos, hormonas y drogas ilícitas (Toro et al. 2016). Se caracterizan por estar en el ambiente en concentraciones de $\mu \mathrm{g} / \mathrm{L}$ o $\mathrm{ng} / \mathrm{L}$ y se relacionan con efectos de toxicidad crónica y disrupción endocrina en los seres vivos (Virkutyte et al. 2010, Kabir et al. 2015).

La presencia de MC en el agua superficial se ha atribuido a la descarga de aguas residuales y a la baja eficiencia de eliminación de los sistemas de tratamiento convencionales (Westerhoff et al. 2005, Chen et al. 2006, Hernando et al. 2006, Zhou et al. 2012). Esto resulta inconveniente para Colombia, donde se estima que sólo el $36 \%$ de las aguas residuales domésticas urbanas son tratadas (DNP 2016).

En sistemas de tratamiento primario avanzado, con procesos físicos y químicos de coagulaciónfloculación, Carballa et al. (2005) reportaron eficiencias de eliminación de aproximadamente $70 \%$ para el diclofenaco y un incremento del $25 \%$ en la eliminación del diazepam y naproxeno, respecto a un sistema convencional. Con bajas eficiencias de eliminación en la carbamazepina y el ibuprofeno.

Los patrones de consumo de los compuestos farmacéuticos por parte de las sociedades, también inciden en la presencia de $\mathrm{MC}$ en el ambiente acuático, especialmente en un país como Colombia donde existe una cultura de automedicación. Sólo en Bogotá D.C. se reveló porcentajes de automedicación del $58 \%$, siendo el paracetamol el compuesto más comercializado (López et al. 2009).

En el agua potable, la presencia de $\mathrm{MC}$ se atribuye a que las plantas de tratamiento de agua potable (PTAP) convencionales no garantizan su completa eliminación por lo que se han reportado compuestos como la iopromida, ibuprofeno, carbamazepina y bisfenol A (BPA) en el agua potable (Westerhoff et al. 2005, Chen et al. 2006, Kim et al. 2007).
Las bajas concentraciones de estos compuestos, el desconocimiento de su presencia y el difícil acceso a las técnicas de medición, han contribuido a que solo unos pocos $\mathrm{MC}$ hayan sido regulados.

El Parlamento Europeo y el Consejo de la Unión Europea (2008), mediante la Directiva 2008/105/ CE establecieron las "Normas de calidad ambiental en el ámbito de la política de aguas" donde se regulan compuestos como el nonilfenol y el octilfenol. El BPA está en revisión para su posible identificación como sustancia prioritaria o peligrosa prioritaria.

En Australia se han desarrollado guías con los límites máximos en el agua superficial para potabilización $(0.35$ a $1050 \mu \mathrm{g} / \mathrm{L})$, donde se incluyen fragancias, fármacos y hormonas (EPHC 2007). Holanda ha propuesto el estándar de calidad de agua superficial destinada para el consumo humano y la cría de peces, donde se incluyó la carbamazepina, metoprolol, metformina y ácido amidotrizoico (Moermond 2014). La USEPA (2016) incluyó en la lista de candidatos a contaminantes y de determinación regulatoria del año 2016, la eritromicina, 17 $\alpha$-estradiol, 17 $\beta$-estradiol, estriol, estronas, $17 \alpha$-etinilestradiol, mestranol y nonilfenol.

En Colombia no hay regulación para compuestos farmacéuticos o disruptores endocrinos (DE) en el medio acuático; sin embargo mediante la Resolución 4143/2012 (MINSALUD 2012), se prohíbe el BPA en los materiales plásticos que entren en contacto con alimentos o bebidas de consumo humano. Aún así, se carece de información científica sobre la presencia de estos MC en las aguas del país.

Tomando en cuenta la escasa información, se desarrolló este estudio donde se buscó analizar la presencia de compuestos farmacéuticos y $\mathrm{DE}$ en el ciclo urbano del agua (CUA), como caso de estudio la ciudad de Santiago de Cali, Colombia.

Esta información es una línea base para futuras investigaciones sobre la evaluación de su posible amenaza, la optimización de los sistemas de potabilización y el tratamiento del agua residual de la 
ciudad, además de la formulación de programas de educación tendientes a mejorar comportamientos de la sociedad hacia la automedicación.

\section{MATERIALES Y MÉTODOS}

\section{Descripción de los sitios de muestreo}

El estudio se desarrolló en la ciudad de Santiago de Cali, siendo la tercera ciudad más poblada de Colombia. El $60 \%$ de la población se abastece del río Cauca a través de la PTAP de Puerto Mallarino (PTAP-PM), la cual tiene un tren de tratamiento con aplicación de carbón activado, desarenación, precloración, coagulación, floculación, sedimentación, filtración, post-cloración y estabilización química con cal.

Después de la captación en la PTAP-PM, el río recibe los efluentes tratados en la planta de tratamiento de aguas residuales de Cañaveralejo (PTAR-C), que trata el $82.7 \%$ de los vertimientos domésticos generados en la ciudad (CONPES 2013), solamente aplicando tratamiento primario avanzado.
El CUA fue monitoreado en ocho puntos (Fig.1): en el agua superficial se tomaron tres puntos sobre el río Cauca: a la entrada de la ciudad (AS-1), en la bocatoma de la PTAP-PM (AS-3) y a la salida de la ciudad (AS-4). Además de un tributario del río llamado Canal Sur (AS-2) que recoge los ríos Cañaveralejo, Melendez y Lili, así como efluentes de aguas residuales sin tratar y lixiviados de un antiguo botadero de la ciudad que se encuentra sellado y clausurado.

Los puntos de agua potable se tomaron en la red de distribución de la PTAP-PM: cerca de la salida de la PTAP (AP-1), en una institución educativa (AP-2) $y$ en una residencia familiar (AP-3). Mientras que el punto de agua residual tratada corresponde al efluente de la PTAR-C (AR-1).

\section{Toma y análisis de muestras}

Se realizaron cinco jornadas de muestreo distribuidas en ocho meses, entre septiembre de 2015 y mayo de 2016, la primera como un muestreo preliminar en el cual se midieron $92 \mathrm{MC}$ (Cuadro I) y cuatro muestreos posteriores para tres grupos selec-

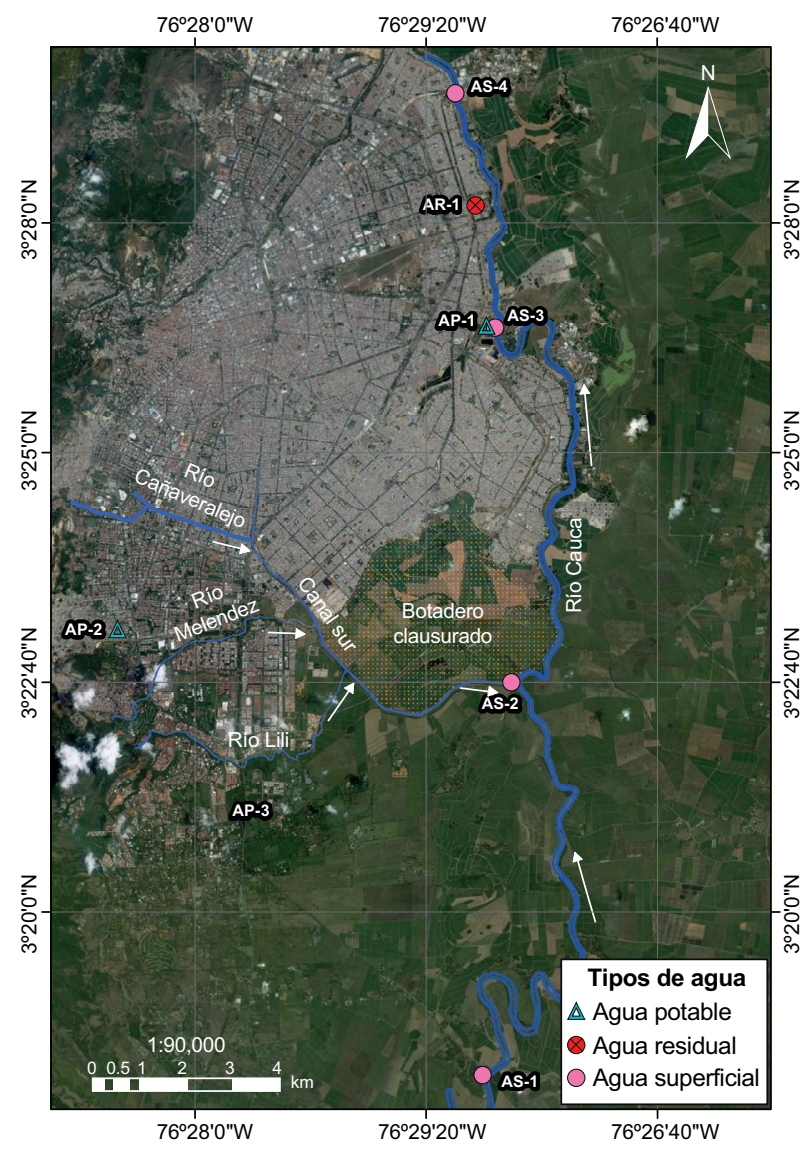

\section{$\underline{\text { Agua superficial }}$}

AS-1: Río Cauca. Entrada Santiago de Cali [3¹8'5.657"N - 76²8'37.121"W]

AS-2: Tributario a Río Cauca. Canal Sur [3²2'39.544"N - 76²8'17,77"W]

AS-3: Río Cauca. Bocatoma planta de potabilización de Puerto Mallarino [3²6'45.496"N - 76²8'29.917"W]

AS-4: Río Cauca. Salida Santiago de Cali [3²9'33.341"N - 76² $\left.28^{\prime} 59.059^{\prime \prime} \mathrm{W}\right]$

Agua potable

AP-1: Red de distribución cercana a planta de potabilización de Puerto Mallarino [ $\left.3^{\circ} 26^{\prime} 45.535^{\prime \prime} \mathrm{N}-76^{\circ} 28^{\prime} 49.77^{\prime \prime} \mathrm{W}\right]$

AP-2: Red de distribución zona intermedia Institución educativa [3²3'43.197"N - 76²32'56.086”W]

AP-3: Red de distribución zona final-Residencia familiar

[321'14.893”N- 76³1'39.906"W]

\section{Agua residual}

AR-1: Efluente planta de tratamiento de aguas residuales de Cañaveralejo [3²8'10.345"N - 76²8'30.593”W]

Fig. 1. Localización de puntos de muestreo en el ciclo urbano del agua de Santiago de Cali 
cionados por presencia, concentración, efectos sobre la biota acuática y presupuesto.

Se tomaron muestras puntuales (los días miércoles, entre las 8:00 y 10:00 am) en recipientes de un litro, conservándolas a $4{ }^{\circ} \mathrm{C}$. Posteriormente, se realizó un proceso de doble filtración mediante filtros de membrana de celulosa de 1.5 y $0.45 \mu \mathrm{m}$ y se envasaron en termos de acero inoxidable. Lo anterior, para conservar la cadena de frío hasta su entrega en el laboratorio Technologiezentrum Wasser (TZW) en Karlsruhe, Alemania.

En el laboratorio TZW, se realizaron los procesos de extracción en fase sólida, seguido de cromatografía líquida acoplado con espectrometría de masas (LC/MS-MS), para el análisis de compuestos farmacéuticos (Vanderford et al. 2003, Locatelli et al. 2011, Wielogórska et al. 2015) y cromatografía de gases acoplado con espectrometría de masas (GC/MS), para el análisis de DE (Durán et al. 2009).

\section{Compuestos cuantificados}

El laboratorio TZW tiene su propia clasificación para estos compuestos, como se observa en el cuadro I, con cinco grupos de compuestos farmacéuticos y un grupo para DE. El grupo I lo conforman $22 \mathrm{MC}$, que incluyen analgésicos, antipiréticos, antiinflamatorios, antirreumáticos, antiepilépticos, agentes hipolipemiantes, tranquilizantes y vasodilatadores.

El grupo II de $18 \mathrm{MC}$, contiene analgésicos, agentes hipolipemiantes, antineoplásicos, beta-bloqueadores, bronquiolíticos y secretolíticos. El grupo III con ocho MC de medios de contraste de rayos X. Dos grupos de antibióticos, el grupo IV con $25 \mathrm{MC}$ y el grupo $\mathrm{V}$ con diez MC. El grupo DE incluye nueve $\mathrm{MC}$ entre hormonas naturales y sintéticas y alquilfenoles.

\footnotetext{
Análisis de la presencia de microcontaminantes

La presencia se evaluó en una escala porcentual, teniendo en cuenta el número de veces que apareció un compuesto en cada uno de los puntos y las jornadas de muestreo. Esto se realizó sólo para los compuestos que tuvieron valores de concentración mayores al límite de detección (LD), sin embargo no se descarta la presencia de los $\mathrm{MC}$ que estuvieron por debajo del LD.

Para los MC reportados en el muestreo preliminar, se evaluó su presencia en los ocho puntos de muestreo y para los grupos seleccionados, se tuvo en cuenta que el mayor número de veces que podría encontrarse el MC es de 40 (ocho puntos por cinco jornadas de muestreo).
}

\section{RESULTADOS Y DISCUSIÓN}

\section{Muestreo preliminar}

Este muestreo arrojó que de los $92 \mathrm{MC}$ cuantificados, 31 estuvieron por encima del LD en el CUA (equivalente a $33.7 \%$ ): 10 del grupo I, tres del grupo II, cinco del grupo III, siete del grupo IV y seis del grupo DE. Los resultados de concentración por punto de muestreo y presencia en el CUA de estos MC se reportan en el cuadro II.

Se observa que los compuestos 10,11-dihydro-10,11-dihidroxicarbamazepina (CBZ-Diol), ibuprofeno, iopromida, iohexol, gemfibrozilo, naproxeno, paracetamol, n-acetil-4-aminoantipirina, sulfametoxazol, trimetoprim, estronas, 4-iso-nonilfenol (4-INF), BPA, n-formil-4-aminoantipirina y dicloxacilina, tuvieron una presencia superior al 50 $\%$. De los cuales los cuatro primeros se encontraron en el agua potable.

Los MC del grupo I que tuvieron las concentraciones más altas fueron paracetamol, naproxeno, gemfibrozilo e ibuprofeno (hasta 200 veces el LD), del grupo II, n-acetil-4-aminoantipirina y n-formil4-aminoantipirina (hasta 13 veces el LD). Por su parte, del grupo III, iohexol, iopromida y ácido diatrizoico (hasta 32 veces el LD), del grupo IV, sulfametoxazol, metronidazol y claritromicina (hasta 12 veces el LD) y del grupo DE 4-INF y BPA (hasta 24 veces el LD).

Con base en los resultados del muestreo preliminar se priorizaron los grupos I, IV y DE para los siguientes cuatro muestreos. El grupo I, tuvo una mayor presencia, así como las concentraciones más altas en el CUA, mismas que estuvieron asociadas con el consumo de medicamentos de venta libre como el ibuprofeno, naproxeno y paracetamol (INVIMA 2016). Algunos de estos compuestos están incluidos en el Plan Obligatorio de Salud (POS) (MINSALUD 2015), lo que favorece su prescripción por parte de las entidades de salud. Entre estos, el gemfibrozilo, que es usado en el tratamiento de colesterol alto, y la carbamazepina, cuyo principal metabolito es el CBZ-Diol, usada para el manejo de epilepsia, neuralgias del nervio trigémino y glosofaríngeas y condiciones de excitación maniaca (Wiklund et al. 1993, Houeto et al. 2012).

Los fármacos del grupo IV como sulfametoxazol, metronidazol y claritromicina se destacaron por su alta concentración. También están incluidos en el POS y son usados para combatir infecciones bacterianas. Adicionalmente, su estudio es relevante debido a que están relacionados con toxicidad crónica en los seres vivos y el desarrollo de resistencia bacteriana (Michael et al. 2013). 
CUADRO I. CLASIFICACIÓN Y LÍMITES DE DETECCIÓN DE COMPUESTOS FARMACÉUTICOS Y DISRUPTORES ENDOCRINOS (DE) REALIZADA POR LOS LABORATORIOS TECHNOLOGIEZENTRUM WASSER, ALEMANIA

\begin{tabular}{|c|c|c|c|c|c|}
\hline \multirow{2}{*}{ Grupo } & \multirow[b]{2}{*}{ Tipo } & \multirow{2}{*}{\multicolumn{2}{|c|}{ Compuestos }} & \multicolumn{2}{|c|}{ Límite de detección (ng/L) } \\
\hline & & & & $\begin{array}{c}\text { Río Cauca y agua } \\
\text { potable }\end{array}$ & $\begin{array}{c}\text { Canal sur y } \\
\text { agua residual }\end{array}$ \\
\hline \multirow{5}{*}{ I } & Antiepilépticos & $\begin{array}{c}\text { 10,11-dihydro- } 10,11- \\
\text { dihidroxicarbamazepina } \\
\text { Carbamazepina }\end{array}$ & $\begin{array}{l}\text { Gabapentina } \\
\text { Lamotrigina } \\
\text { Primidona } \\
\end{array}$ & \multirow{4}{*}{$<10$} & \multirow{4}{*}{$<50$} \\
\hline & $\begin{array}{l}\text { Agentes hipolipe- } \\
\text { miantes }\end{array}$ & $\begin{array}{l}\text { Bezafibrato } \\
\text { Ácido clofíbrico } \\
\text { Etofibrato } \\
\end{array}$ & $\begin{array}{l}\text { Fenofibrato } \\
\text { Ácido fenofíbrico } \\
\text { Gemfibrozilo }\end{array}$ & & \\
\hline & $\begin{array}{l}\text { Analgésicos, } \\
\text { Antipiréticos, } \\
\text { Antiinflamatorios, } \\
\text { Antirreumáticos }\end{array}$ & $\begin{array}{l}\text { Diclofenaco } \\
\text { Fenoprofeno } \\
\text { Ibuprofeno } \\
\text { Indometacina }\end{array}$ & $\begin{array}{l}\text { Ketoprofeno } \\
\text { Naproxeno } \\
\text { Paracetamol }\end{array}$ & & \\
\hline & Tranquilizantes & $\begin{array}{l}\text { Diazepam } \\
\text { Oxazepam }\end{array}$ & Venlafaxina & & \\
\hline & Vasodilatadores & Pent & & $<10$ & $<50$ \\
\hline \multirow{5}{*}{ II } & $\begin{array}{c}\text { Agentes } \\
\text { hipolipemiantes }\end{array}$ & Sim & & \multirow[b]{2}{*}{$<10$} & \multirow[b]{2}{*}{$<50$} \\
\hline & Analgésicos & $\begin{array}{c}\text { Dimetilaminofenazona } \\
\text { N-acetil-4-aminoantipirina } \\
\text { N-formil-4-aminoantipirina }\end{array}$ & $\begin{array}{c}\text { Fenazona } \\
\text { Propifenazona }\end{array}$ & & \\
\hline & Antineoplásicos & Ciclofosfamida & Ifosfamida & \multirow{3}{*}{$<10$} & \multirow{3}{*}{$<50$} \\
\hline & Beta-bloqueadores & $\begin{array}{l}\text { Atenolol } \\
\text { Betaxolol } \\
\text { Bisoprolol } \\
\text { Metoprolol } \\
\end{array}$ & $\begin{array}{l}\text { Pindolol } \\
\text { Propranolol } \\
\text { Sotalol }\end{array}$ & & \\
\hline & $\begin{array}{l}\text { Bronquiolítico } \\
\text { Secretolítico } \\
\end{array}$ & $\begin{array}{l}\text { Clenbuterol } \\
\text { Salbutamol }\end{array}$ & Terbutalin & & \\
\hline III & $\begin{array}{l}\text { Medios de contraste } \\
\text { de rayos X }\end{array}$ & $\begin{array}{c}\text { Ácido diatrizoico } \\
\text { Iohexol } \\
\text { Iomeprol } \\
\text { Iopamidol } \\
\end{array}$ & $\begin{array}{c}\text { Iopromida } \\
\text { Ácido Iotalámico } \\
\text { Ioversol } \\
\text { Ácido Ioxitalamico }\end{array}$ & $<10$ & $<50$ \\
\hline \multirow{3}{*}{ IV } & Antibióticos & $\begin{array}{l}\text { Amoxicilina } \\
\text { Cloxacilina } \\
\text { Dicloxacilina } \\
\text { Nafcilina } \\
\end{array}$ & $\begin{array}{l}\text { Oxacilina } \\
\text { Penicilina G } \\
\text { Penicilina V }\end{array}$ & $<20$ & $<100$ \\
\hline & & $\begin{array}{c}\text { Cloranfenicol } \\
\text { Claritromicina } \\
\text { Dapsona } \\
\text { Dehidrato-eritromicina A } \\
\text { Eritromicina A } \\
\text { Furazolidon } \\
\text { Metronidazol } \\
\text { Oleandomicina } \\
\text { Ronidazol } \\
\end{array}$ & $\begin{array}{c}\text { Roxitromicina } \\
\text { Espiramicina } \\
\text { Sulfadiazina } \\
\text { Sulfadimidina } \\
\text { Sulfamerazina } \\
\text { Sulfametoxazol } \\
\text { Tilosina } \\
\text { Virginiamicina }\end{array}$ & $<10$ & $<50$ \\
\hline & & \multicolumn{2}{|c|}{ Trimetoprim } & $<5$ & $<25$ \\
\hline \multirow{5}{*}{ DE } & Antibióticos & $\begin{array}{l}\text { Clortetraciclina } \\
\text { Ciprofloxacina } \\
\text { Doxiciclina } \\
\text { Enoxacina } \\
\text { Enrofloxacina } \\
\end{array}$ & $\begin{array}{l}\text { Meclociclina } \\
\text { Norfloxacina } \\
\text { Ofloxacina } \\
\text { Oxitetraciclina } \\
\text { Tetraciclina }\end{array}$ & $<20$ & $<100$ \\
\hline & \multirow[t]{2}{*}{$\begin{array}{c}\text { Hormonas naturales } \\
\text { y sintéticas }\end{array}$} & $\begin{array}{l}\text { 17ß-estradiol } \\
\text { Estronas }\end{array}$ & $17 \alpha$-etinilestradiol & $<0.2$ & $<0.5$ \\
\hline & & $\begin{array}{c}\text { Estriol } \\
\text { Mestranol }\end{array}$ & Noretisterona & $<2$ & $<5$ \\
\hline & Alquilfenoles & \multicolumn{2}{|c|}{ 4-iso-nonilfenol } & $<50$ & $<130$ \\
\hline & & 4-tert-octilfenol & Bisfenol A & $<10$ & $<25$ \\
\hline
\end{tabular}


CUADRO II. CONCENTRACIÓN (ng/L) Y PRESENCIA DE MICROCONTAMINANTES EN MUESTREO PRELIMINAR

\begin{tabular}{|c|c|c|c|c|c|c|c|c|c|c|}
\hline \multirow{2}{*}{ Grupo } & \multirow{2}{*}{ Compuesto } & \multicolumn{4}{|c|}{$\begin{array}{l}\text { Agua superficial } \\
\text { (AS) }\end{array}$} & \multicolumn{3}{|c|}{$\begin{array}{l}\text { Agua potable } \\
\text { (AP) }\end{array}$} & \multirow{2}{*}{$\begin{array}{c}\begin{array}{c}\text { Agua residual } \\
\text { (AR) }\end{array} \\
\text { AR-1 }\end{array}$} & \multirow{2}{*}{$\begin{array}{c}\text { Presencia * } \\
\text { CUA }(\%)\end{array}$} \\
\hline & & AS-1 & AS-2 & AS-3 & AS-4 & AP-1 & AP-2 & AP-3 & & \\
\hline \multirow{10}{*}{ I } & $\begin{array}{l}\text { 10,11-dihydro-10,11- } \\
\text { dihidroxicarbamazepina }\end{array}$ & 18 & 470 & 21 & 110 & 19 & 24 & 25 & 910 & 100 \\
\hline & Ibuprofeno & 12 & 1500 & 23 & 360 & 17 & 16 & 16 & 3100 & 100 \\
\hline & Gemfibrozilo & 51 & 1500 & 26 & 250 & $<\mathrm{LD}$ & $<\mathrm{LD}$ & $<\mathrm{LD}$ & 4000 & 62.5 \\
\hline & Naproxeno & 34 & 1900 & 34 & 630 & $<\mathrm{LD}$ & $<\mathrm{LD}$ & $<\mathrm{LD}$ & 4900 & 62.5 \\
\hline & Paracetamol & 28 & 11000 & 13 & 1700 & $<\mathrm{LD}$ & $<\mathrm{LD}$ & $<\mathrm{LD}$ & 520 & 62.5 \\
\hline & Carbamazepina & $<\mathrm{LD}$ & 81 & $<\mathrm{LD}$ & 17 & $<\mathrm{LD}$ & $<\mathrm{LD}$ & $<\mathrm{LD}$ & 140 & 37.5 \\
\hline & Diclofenaco & $<\mathrm{LD}$ & 92 & $<\mathrm{LD}$ & 27 & $<\mathrm{LD}$ & $<\mathrm{LD}$ & $<\mathrm{LD}$ & 240 & 37.5 \\
\hline & Gabapentina & $<\mathrm{LD}$ & 310 & $<\mathrm{LD}$ & 48 & $<\mathrm{LD}$ & $<\mathrm{LD}$ & $<\mathrm{LD}$ & 400 & 37.5 \\
\hline & Ácido fenofíbrico & $<\mathrm{LD}$ & $<\mathrm{LD}$ & $<\mathrm{LD}$ & 14 & $<\mathrm{LD}$ & $<\mathrm{LD}$ & $<\mathrm{LD}$ & 190 & 25.0 \\
\hline & Ketoprofeno & $<\mathrm{LD}$ & $<\mathrm{LD}$ & $<\mathrm{LD}$ & $<\mathrm{LD}$ & $<\mathrm{LD}$ & $<\mathrm{LD}$ & $<\mathrm{LD}$ & 75 & 12.5 \\
\hline \multirow{3}{*}{ II } & N-acetil-4-aminoantipirina & 62 & 650 & 37 & 100 & $<\mathrm{LD}$ & $<\mathrm{LD}$ & $<\mathrm{LD}$ & 1.1 & 62.5 \\
\hline & $\mathrm{N}$-formil-4-aminoantipirina & 21 & 170 & $<\mathrm{LD}$ & 33 & $<\mathrm{LD}$ & $<\mathrm{LD}$ & $<\mathrm{LD}$ & 350 & 50.0 \\
\hline & Metoprolol & $<\mathrm{LD}$ & 69 & $<\mathrm{LD}$ & 15 & $<\mathrm{LD}$ & $<\mathrm{LD}$ & $<\mathrm{LD}$ & 16 & 37.5 \\
\hline \multirow{5}{*}{ III } & Iopromida & 74 & 1.5 & 82 & 320 & 70 & 69 & 66 & 2.1 & 100 \\
\hline & Iohexol & $<\mathrm{LD}$ & 2.3 & 21 & 180 & 35 & 15 & 26 & 1 & 87.5 \\
\hline & Ácido diatrizoico & $<\mathrm{LD}$ & 930 & $<\mathrm{LD}$ & 35 & $<\mathrm{LD}$ & $<\mathrm{LD}$ & $<\mathrm{LD}$ & 420 & 37.5 \\
\hline & Iopamidol & $<\mathrm{LD}$ & 60 & $<\mathrm{LD}$ & 11 & $<\mathrm{LD}$ & $<\mathrm{LD}$ & $<\mathrm{LD}$ & 74 & 37.5 \\
\hline & Ácido iotalamico & $<\mathrm{LD}$ & 120 & $<\mathrm{LD}$ & $<\mathrm{LD}$ & $<\mathrm{LD}$ & $<\mathrm{LD}$ & $<\mathrm{LD}$ & 80 & 25.0 \\
\hline \multirow{7}{*}{ IV } & Sulfametoxazol & 12 & 440 & 15 & 68 & $<\mathrm{LD}$ & $<\mathrm{LD}$ & $<\mathrm{LD}$ & 580 & 62.5 \\
\hline & Trimetoprim & 6 & 100 & 6 & 28 & $<\mathrm{LD}$ & $<\mathrm{LD}$ & $<\mathrm{LD}$ & 220 & 62.5 \\
\hline & Dicloxacilina & 23 & $<\mathrm{LD}$ & 24 & 150 & $<\mathrm{LD}$ & $<\mathrm{LD}$ & $<\mathrm{LD}$ & 190 & 50.0 \\
\hline & Claritromicina & $<\mathrm{LD}$ & 65 & $<\mathrm{LD}$ & 24 & $<\mathrm{LD}$ & $<\mathrm{LD}$ & $<\mathrm{LD}$ & 430 & 37.5 \\
\hline & Metronidazol & $<\mathrm{LD}$ & 50 & $<\mathrm{LD}$ & 36 & $<\mathrm{LD}$ & $<\mathrm{LD}$ & $<\mathrm{LD}$ & 510 & 37.5 \\
\hline & Dehidrato-erithromicina A & $<\mathrm{LD}$ & $<\mathrm{LD}$ & $<\mathrm{LD}$ & $<\mathrm{LD}$ & $<\mathrm{LD}$ & $<\mathrm{LD}$ & $<\mathrm{LD}$ & 99 & 12.5 \\
\hline & Tilosina & $<\mathrm{LD}$ & $<\mathrm{LD}$ & $<\mathrm{LD}$ & $<\mathrm{LD}$ & $<\mathrm{LD}$ & $<\mathrm{LD}$ & $<\mathrm{LD}$ & 89 & 12.5 \\
\hline \multirow{6}{*}{$\mathrm{DE}$} & Estronas & 1.0 & 40 & 1.5 & 6.4 & $<\mathrm{LD}$ & $<\mathrm{LD}$ & $<\mathrm{LD}$ & 13 & 62.5 \\
\hline & 4-iso-nonilfenol & 70 & 2400 & 56 & 190 & $<\mathrm{LD}$ & $<\mathrm{LD}$ & $<\mathrm{LD}$ & 1200 & 62.5 \\
\hline & Bisfenol A & 23 & 420 & 17 & 63 & $<\mathrm{LD}$ & $<\mathrm{LD}$ & $<\mathrm{LD}$ & 410 & 62.5 \\
\hline & Estriol & $<\mathrm{LD}$ & 47 & $<\mathrm{LD}$ & 6.0 & $<\mathrm{LD}$ & $<\mathrm{LD}$ & $<\mathrm{LD}$ & 97 & 37.5 \\
\hline & 4-tert-octilfenol & $<\mathrm{LD}$ & 33 & $<\mathrm{LD}$ & $<\mathrm{LD}$ & $<\mathrm{LD}$ & $<\mathrm{LD}$ & 15 & 34 & 37.5 \\
\hline & $17 \beta$-estradiol & $<\mathrm{LD}$ & 8.7 & $<\mathrm{LD}$ & $<\mathrm{LD}$ & $<\mathrm{LD}$ & $<\mathrm{LD}$ & $<\mathrm{LD}$ & $<\mathrm{LD}$ & 12.5 \\
\hline
\end{tabular}

* Una presencia del $100 \%$ equivale a que los microcontaminantes se identificaron por encima del límite de detección (LD) en ocho puntos de muestreo. CUA = ciclo urbano del agua, $\mathrm{DE}=$ disruptores endocrinos

El grupo DE se caracterizó por la concentración de compuestos como 4-INF, BPA y estronas. Este grupo ha llamado la atención de los investigadores por sus efectos tóxicos sobre la vida acuática, actividad estrogénica a pocos $\mu \mathrm{g} / \mathrm{L}$, anomalías en el sistema reproductivo de animales e inducción de la síntesis de vitelogenina en peces (Wenzel et al. 2004, Kumar y Xagoraraki 2010), lo que motiva el estudio sobre su presencia y posibles efectos en la región.

\author{
Presencia y concentración de grupos seleccionados \\ (I, IV, DE) \\ El conjunto de MC medidos en los grupos I, IV \\ y DE fue de 56, de los cuales 24 presentaron con- \\ centraciones superiores al LD, equivalente a $42.9 \%$ : \\ 10 del grupo I, ocho del grupo IV y seis del grupo \\ DE. En la figura 2 se observa el comportamiento de \\ los MC a lo largo del río Cauca y el aporte de sus \\ principales tributarios.
}




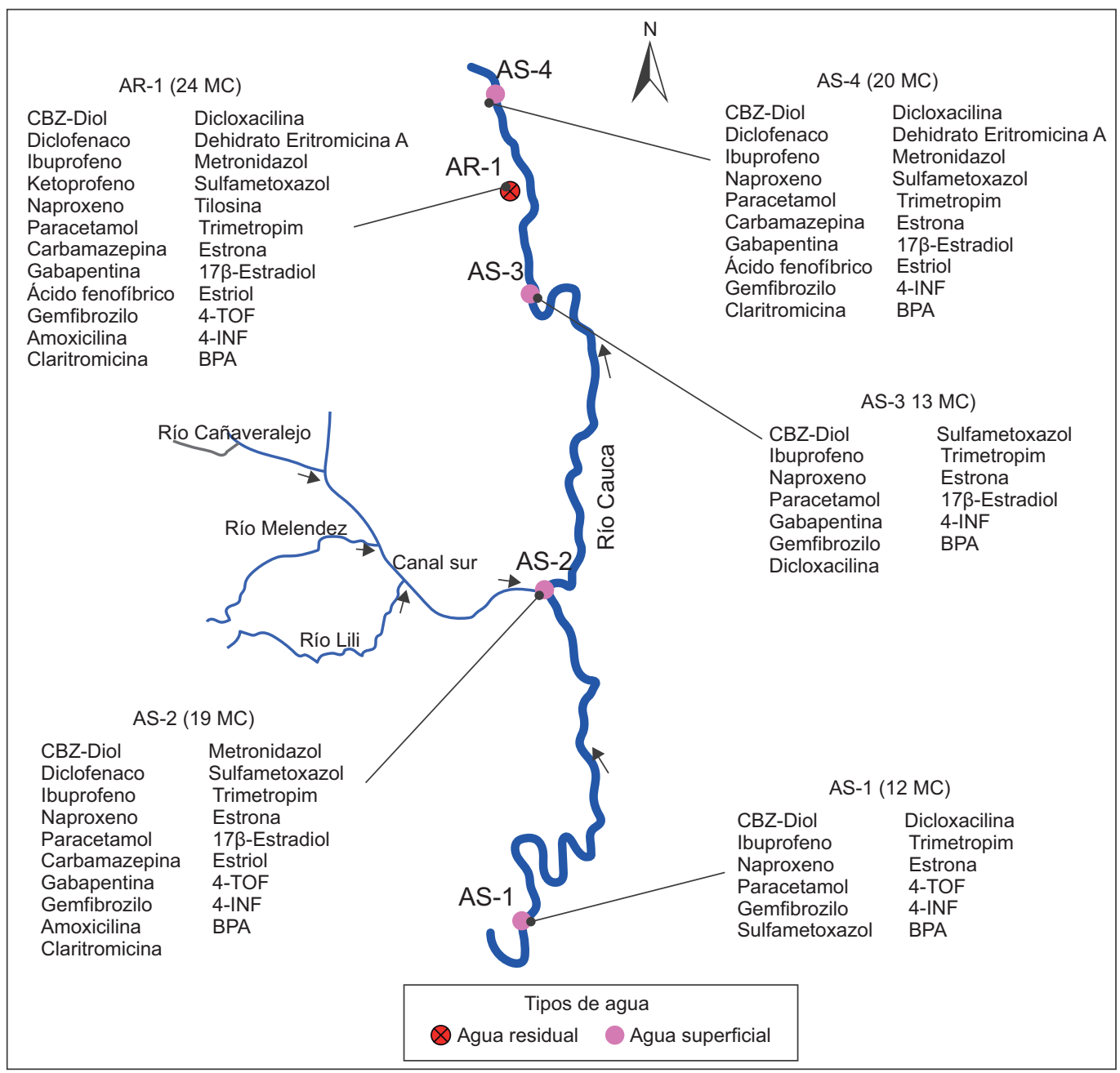

Fig. 2. Cantidad de microcontaminantes encontrados por encima del límite de detección en los puntos del río Cauca y sus principales tributarios. CBZ-Diol $=10,11$-dihydro-10,11-dihidroxicarbamazepina, 4-TOF = 4-tert-octilfenol, 4-INF = 4-iso-nonilfenol, BPA = bisfenol A

En AS-1, el río contiene $12 \mathrm{MC}$, posiblemente asociado a vertimientos domésticos e industriales aguas arriba. En el siguiente punto sobre el río, en AS-3, la presencia se incrementa a $13 \mathrm{MC}$ siendo la gabapentina y $17 \beta$-estradiol los dos nuevos MC, que se infiere fueron aportados por AS-2, mientras que el 4-tert-octilfenol (4-TOF) se redujo a valores menores al LD.

En AS-4, la presencia fue de $20 \mathrm{MC}$, siendo la carbamazepina, diclofenaco, ácido fenofíbrico, claritromicina, dehidrato eritromicina A, metronidazol y estriol los nuevos MC encontrados, en comparación con AS-3, lo cual se asocia a la descarga de PTAR-C. Esto permite afirmar que la ciudad de Santiago de Cali constituye un aportante de $\mathrm{MC}$ al río Cauca, aumentando la potencial amenaza ambiental.

En los puntos de agua potable se detectó la presencia de seis MC por encima del LD: CBZ-Diol, carbamazepina, ibuprofeno, estronas, estriol y 4-TOF. De los cuales se reportó una mayor concentración en el agua potable que en la entrada de la PTAP-PM (AS-3), para la carbamazepina, el estriol y el 4-TOF (Cuadro III).

Estas variaciones en el agua potable se asocian a la incertidumbre en la técnica analítica, para las concentraciones cercanas al LD, por lo que se consideran prácticamente iguales. También por un posible incremento de la concentración en AS-3 antes de tomar la muestra para este estudio que, por ser exploratorio, 
no tuvo en cuenta los tiempos de viaje del agua al interior de la PTAP-PM.

Las eficiencias de eliminación en la PTAP-PM fueron de $0 \%$ para el CBZ-Diol y las estronas. Para el CBZ-Diol, este resultado se asocia con sus características hidrofílicas, recalcitrantes, su difícil mineralización y la tendencia a acumularse en exceso de cloro (Zhang et al. 2008, Fenet et al. 2012, Soufan et al. 2013), lo que favoreció su paso por la PTAP-PM sin variar su concentración.

Las estronas, sólo se presentaron una vez en los tres puntos de agua potable, lo que se relaciona también con su moderada hidrofilicidad, debido a un coeficiente de partición octanol-agua (log Kow) de 3.13; aunque no se descarta que su presencia esté asociada también con la incertidumbre de la técnica analítica empleada.

El ibuprofeno tuvo una eficiencia de eliminación promedio en la PTAP-PM de $28.3 \%$ y estuvo presente en el agua potable en cuatro de los cinco muestreos. Estudios previos han reportado que este MC tiene bajas eficiencias de eliminación ante procesos como coagulación/floculación, cloración y adsorción con carbón activado (Westerhoff et al. 2005, Kleywegt et al. 2011, Simazaki et al.2015), los cuales se llevan a cabo en la PTAP-PM.

El paracetamol siempre estuvo bajo el LD en el agua potable, a pesar de ser un compuesto altamente hidrofílico (log Kow de 0.46 ) y de tener la concentración más alta en AS-3, con respecto a otros MC. De acuerdo con lo reportado por Westerhoff et al. (2005), quienes trabajaron a escala de laboratorio, su eliminación se puede atribuir a los procesos de carbón activado y oxidación con cloro, donde los autores reportaron eficiencias del 69 y $95 \%$, respectivamente.

En otros estudios, Snyder et al. (2007) encontraron eficiencias del $52 \%$ con carbón activado granular para la eliminación del paracetamol. Mientras que Bedner y MacCrehan (2006), identificaron que a una dosis de cloro de $4 \mathrm{ppm}$, el $88 \%$ del paracetamol se transformó en n-acetil-p-benzoquinona y p-benzoquinona, disminuyendo la presencia del compuesto en su forma original.

Del cuadro III se observa que las concentraciones en los puntos sobre el río Cauca se incrementaron en el sentido del flujo, siendo hasta cinco veces más altas en AS-3 con respecto a AS-1 y hasta siete veces más altas en AS-4 que en AS-3.

El primer tramo, entre AS-1 y AS-3, está influenciado por la descarga de AS-2 y el segundo tramo, entre AS-3 y AS-4, por AR-1 (Fig. 2), por lo que el mayor efecto se atribuye al punto AR-1 donde las concentraciones son de hasta ocho veces más altas que en AS-2, lo que puede incidir en la capacidad de autodepuración del río Cauca.

Por concentración y presencia en el CUA de Santiago de Cali se destacan los siguientes compuestos: paracetamol, CBZ-Diol, ibuprofeno, naproxeno, gemfibrozilo, sulfametoxazol, estronas, 4-INF y BPA. El paracetamol fue el que tuvo concentraciones de hasta 10 veces más altas en el agua superficial y residual.

La presencia del paracetamol se relaciona con la propagación de enfermedades como el zika, chikungunya y dengue, comunes en zonas tropicales y transmitidas por el mosquito Aedes aegypti, dado que es el medicamento normalmente prescrito para el manejo de la fiebre y el dolor.

De acuerdo con el informe epidemiológico del municipio de Santiago de Cali, entre 2015 y primer semestre de 2016, se notificaron 33430 casos de dengue, 13837 de zika y 2682 de chikungunya (Alcaldía Santiago de Cali 2015, 2016), condición que disparó el consumo del paracetamol.

La presencia de los demás MC puede asociarse también al consumo por parte de la población, sin embargo hasta el momento no hay una evidencia clara sobre su procedencia. No obstante, por sus características físicas y químicas, Koumaki et al. (2017) indican que en el agua superficial la eliminación de los $\mathrm{MC}$ está dada por procesos como hidrólisis, volatilización, adsorción, fotólisis y biodegradación. Dichos procesos pueden transformar los MC en cuestión de horas o días, incidiendo sobre su presencia.

El metabolito CBZ-Diol fue uno de los MC más persistentes. Se estima que el $28 \%$ de la carbamazepina se excreta sin sufrir alteración a través de las heces, el $72 \%$ restante es absorbido por el cuerpo y metabolizado hasta CBZ-Diol, que luego es eliminado por la orina en un $30 \%$ del compuesto original (Zhang et al. 2008, Houeto et al. 2012).

Lo anterior, aunado a que el CBZ-Diol es también un metabolito de la oxcarbamazapina, derivado de la carbamazapina (Miao y Metcalfe 2003), favorece su presencia por encima de la carbamazepina. Condición que se confirmó en este estudio donde se encontró que tanto en la PTAR-C como en el agua superficial, la concentración del metabolito fue aproximadamente seis veces mayor que la de la carbamazepina.

Koumaki et al. (2017) consideran a la biodegradación como el principal mecanismo de eliminación del ibuprofeno en el agua superficial, con mayor eficiencia bajo condiciones aerobias, pues la tasa de eliminación disminuye cuando baja la concentración de oxígeno disuelto. Sin embargo, en el río Cauca 
CUADRO III. CONCENTRACIÓN MEDIA (ng/L) Y PRESENCIA DE MICROCONTAMINANTES ENCONTRADOS POR ENCIMA DEL LÍMITE DE DETECCIÓN (LD)

\begin{tabular}{|c|c|c|c|c|c|c|c|c|c|c|}
\hline \multirow[t]{2}{*}{ Grupo } & \multirow[t]{2}{*}{ Compuestos } & \multicolumn{4}{|c|}{ Agua superficial (AS) } & \multicolumn{3}{|c|}{ Agua potable (AP) } & \multirow{2}{*}{$\begin{array}{c}\begin{array}{c}\text { Agua residual } \\
(\mathrm{AR})\end{array} \\
\text { AR-1 }\end{array}$} & \multirow{2}{*}{$\begin{array}{c}\text { Presencia * } \\
\text { CUA }(\%)\end{array}$} \\
\hline & & AS-1 & AS-2 & AS-3 & AS-4 & AP-1 & AP-2 & AP-3 & & \\
\hline \multirow{10}{*}{ I } & Ibuprofeno & 13.5 & 894 & 32.8 & 229 & 23.5 & 19.3 & 19.5 & 2720 & 88 \\
\hline & $\begin{array}{l}\text { 10,11-dihydro-10,11- } \\
\text { dihidroxicarbamazepina }\end{array}$ & 21.0 & 290 & 26.3 & 77.0 & 26.5 & 24.3 & 28.0 & 818 & 83 \\
\hline & Naproxeno & 30.6 & 1210 & 58.2 & 411 & $<\mathrm{LD}$ & $<\mathrm{LD}$ & $<\mathrm{LD}$ & 5840 & 63 \\
\hline & Gemfibrozilo & 24.8 & 1014 & 53.5 & 234 & $<\mathrm{LD}$ & $<\mathrm{LD}$ & $<\mathrm{LD}$ & 4820 & 58 \\
\hline & Paracetamol & 48.8 & 6820 & 226 & 1670 & $<\mathrm{LD}$ & $<\mathrm{LD}$ & $<\mathrm{LD}$ & 13555 & 55 \\
\hline & Gabapentina & $<\mathrm{LD}$ & 191 & 14.0 & 37.3 & $<\mathrm{LD}$ & $<\mathrm{LD}$ & $<\mathrm{LD}$ & 338 & 35 \\
\hline & Carbamazepina & $<\mathrm{LD}$ & 64.0 & $<\mathrm{LD}$ & 16.0 & 15.0 & $<\mathrm{LD}$ & $<\mathrm{LD}$ & 132 & 33 \\
\hline & Diclofenaco & $<\mathrm{LD}$ & 111 & $<\mathrm{LD}$ & 39.8 & $<\mathrm{LD}$ & $<\mathrm{LD}$ & $<\mathrm{LD}$ & 184 & 28 \\
\hline & Ácido Fenofíbrico & $<\mathrm{LD}$ & $<\mathrm{LD}$ & $<\mathrm{LD}$ & 14.0 & $<\mathrm{LD}$ & $<\mathrm{LD}$ & $<\mathrm{LD}$ & 124 & 15 \\
\hline & Ketoprofeno & $<\mathrm{LD}$ & $<\mathrm{LD}$ & $<\mathrm{LD}$ & $<\mathrm{LD}$ & $<\mathrm{LD}$ & $<\mathrm{LD}$ & $<\mathrm{LD}$ & 82.3 & 8.0 \\
\hline \multirow{8}{*}{ IV } & Sulfametoxazol & 14.5 & 246 & 20.0 & 58.3 & $<\mathrm{LD}$ & $<\mathrm{LD}$ & $<\mathrm{LD}$ & 518 & 48 \\
\hline & Metronidazol & $<\mathrm{LD}$ & 94.4 & $<\mathrm{LD}$ & 29.0 & $<\mathrm{LD}$ & $<\mathrm{LD}$ & $<\mathrm{LD}$ & 748 & 38 \\
\hline & Trimetoprim & 6.0 & 61.8 & 6.0 & 19.5 & $<\mathrm{LD}$ & $<\mathrm{LD}$ & $<\mathrm{LD}$ & 202 & 38 \\
\hline & Claritromicina & $<\mathrm{LD}$ & 60.0 & $<\mathrm{LD}$ & 18.5 & $<\mathrm{LD}$ & $<\mathrm{LD}$ & $<\mathrm{LD}$ & 263 & 23 \\
\hline & Dicloxacilina & 23.0 & $<\mathrm{LD}$ & 24.0 & 150 & $<\mathrm{LD}$ & $<\mathrm{LD}$ & $<\mathrm{LD}$ & 190 & 10 \\
\hline & Dehidrato Eritromicina A & $<\mathrm{LD}$ & $<\mathrm{LD}$ & $<\mathrm{LD}$ & 12.0 & $<\mathrm{LD}$ & $<\mathrm{LD}$ & $<\mathrm{LD}$ & 91.0 & 8.0 \\
\hline & Amoxicilina & $<\mathrm{LD}$ & 33.0 & $<\mathrm{LD}$ & $<\mathrm{LD}$ & $<\mathrm{LD}$ & $<\mathrm{LD}$ & $<\mathrm{LD}$ & 160 & 5.0 \\
\hline & Tilosina & $<\mathrm{LD}$ & $<\mathrm{LD}$ & $<\mathrm{LD}$ & $<\mathrm{LD}$ & $<\mathrm{LD}$ & $<\mathrm{LD}$ & $<\mathrm{LD}$ & 89.0 & 3.0 \\
\hline \multirow{6}{*}{ DE } & Estronas & 2.1 & 13.8 & 1.0 & 2.9 & 1.4 & 1.3 & 3.6 & 13.3 & 60 \\
\hline & Estriol & $<\mathrm{LD}$ & 39.7 & $<\mathrm{LD}$ & 6.0 & $<\mathrm{LD}$ & $<\mathrm{LD}$ & 19.0 & 92.6 & 25 \\
\hline & $17 \beta$ Estradiol & $<\mathrm{LD}$ & 22.9 & 0.2 & $<\mathrm{LD}$ & $<\mathrm{LD}$ & $<\mathrm{LD}$ & $<\mathrm{LD}$ & 6.4 & 13 \\
\hline & 4-iso-nonilfenol & 143 & 935 & 66.3 & 153 & $<\mathrm{LD}$ & $<\mathrm{LD}$ & $<\mathrm{LD}$ & 988 & 55 \\
\hline & 4-tert-octilfenol & 30.0 & 29.5 & $<\mathrm{LD}$ & 14.0 & $<\mathrm{LD}$ & $<\mathrm{LD}$ & 15.0 & 136 & 23 \\
\hline & Bisfenol A & 27.4 & 319 & 24.4 & 65.6 & $<\mathrm{LD}$ & $<\mathrm{LD}$ & $<\mathrm{LD}$ & 626 & 63 \\
\hline
\end{tabular}

*Una presencia del $100 \%$ equivale a que los microcontaminantes se identificaron por encima del límite de detección en ocho puntos durante las cinco jornadas de muestreo. $\mathrm{CUA}=$ ciclo urbano del agua, $\mathrm{DE}=$ disruptores endocrinos

esta biodegradación puede verse afectada debido a la continua descarga de este MC y a que la concentración de oxígeno disuelto ha llegado por debajo de $2 \mathrm{mg} / \mathrm{L}$ (Pérez et al. 2012).

El naproxeno tiene baja biodegradabilidad en el agua superficial y una vida media estimada en 27 días (Grenni et al. 2013). De acuerdo con Koumaki et al. (2017), el principal mecanismo de eliminación del naproxeno es la fotólisis, en menos de 24 horas. Sin embargo, se debe considerar que el río Cauca ha tenido eventos de alta turbiedad con valores máximos entre 476 y 1130 UNT (Montoya et al. 2013), que interfieren con el paso de luz solar afectando dicha fotodegradación.

El gemfibrozilo es un compuesto hidrofóbico con un $\log$ Kow de 4.77, por lo que tiende a fijarse mejor en el suelo y el sedimento. Se considera persistente por su baja biodegradabilidad con una vida media estimada en 70 días (Grenni et al. 2013), condición que favorece su presencia en el agua superficial.

El sulfametoxazol es un antibiótico del grupo de las sulfonamidas, usado en medicina humana y veterinaria. Se estima que entre el 15 y el $25 \%$ es excretado sin ser metabolizado por el cuerpo humano y animal (Radke et al. 2009), por lo que es un compuesto ubicuo en el medio acuático debido a su carácter hidrofílico (log Kow de 0.89).

Aunque el sulfametoxazol se considera persistente, se ha encontrado que bacterias de la especie Bacillus firmus y Bacillus cereus tienen potencial de degradarlo con alta eficiencia en ríos (Su et al. 2016). La fotodegradación es su principal mecanismo de degradación (Xu et al. 2011), sin embargo esta puede verse afectada por los altos valores de turbiedad en el río Cauca.

Las estronas se encontraron en bajas concentraciones con respecto a los demás MC, pero se consideran importantes por ser de los compuestos con mayor 
potencial de disrupción endocrina (Jürgens et al. 2002, Sweeney et al. 2015). Presentan baja volatilidad y biodegradabilidad (Ying et al. 2002), lo cual puede favorecer su presencia en el agua superficial.

De los MC analizados, el 4-INF tuvo la concentración más alta a la entrada de la ciudad, para luego reducirse a poco menos de la mitad en AS-3. Esta reducción podría asociarse a sus características altamente hidrofóbicas ( $\log$ Kow de 5.76), que favorecen su adsorción en los sedimentos, removiéndolo del agua superficial, pues se considera poco biodegradable y por ende persistente (Xu et al. 2006, Ömeroğlu y Sanin 2014).

El BPA tiene una vida media corta, rápida biodegradabilidad y modera hidrofobicidad (log Kow de 3.32 ), por lo que puede ser adsorbido por el material orgánico del suelo y el sedimento (Peng et al. 2015). Sin embargo, es uno de los MC más comunes en el agua residual, lo que le confiere alta ubicuidad y lo convierte en pseudopersistente (Flint et al. 2012). Esto evidenció en AR-1 donde la concentración media fue 16 veces mayor al promedio en el río Cauca.

La presencia de estos MC en el CUA de la ciudad de Santiago de Cali constituye una alerta sobre su posible amenaza ecológica o a la salud humana. El gemfibrozilo presentó genotoxicidad en peces a una concentración de $380 \mathrm{ng} / \mathrm{L}$ (Rocco et al. 2012), concentración cuatro veces menor a la encontrada en AS-2.

Estudios sobre el ibuprofeno reportan que es sospechoso de influenciar en la ruta de la ciclooxigenasa $(\mathrm{COX})$ y la inducción de vitelogenina en peces machos. Además de retraso en la eclosión de huevos de peces a una concentración de $100 \mathrm{ng} / \mathrm{L}$ (Flippin et al. 2007, Han et al. 2010), valor 15 veces menor al encontrado en AS- 2 y cuatro veces menor al de AS-4.

Los DE, se conocen por generar actividad estrogénica, alteraciones en funciones reproductivas y feminización en peces (Wenzel et al. 2004, Kumar y Xagoraraki 2010). En los seres humanos pueden tener efectos como pubertad temprana, cáncer de seno, de próstata, entre otras anomalías genéticas (Parent et al. 2015, Castro y Fontoura 2015, Burks et al. 2016), por lo que están siendo considerados para regulación.

Aunque estudios previos han reportado que la concentración de $\mathrm{MC}$ en el agua potable no genera un riesgo para la salud humana (Schwab et al. 2005, Mompelat et al. 2009, de Jesus Gaffney et al. 2015, Simazaki et al. 2015), se considera importante realizar estudios de mayor complejidad y duración que permitan contar con evidencias científicas sobre los riesgos a la salud en la zona.

\section{CONCLUSIONES}

Se detectó la presencia de MC en el CUA de la ciudad de Santiago de Cali, con los puntos más críticos en el tributario Canal Sur, a la salida de la ciudad y el efluente de la PTAR-C. Lo cual indica que la ciudad genera un aporte importante de estos compuestos sobre el río Cauca, siendo el CBZ-Diol, ibuprofeno, gemfibrozilo, naproxeno, paracetamol, sulfametoxazol, las estronas, 4-INF y BPA, los prioritarios por su presencia y concentración. Esta presencia pone en evidencia la posible amenaza sobre la biota acuática del río Cauca y la población caleña que se abastece de esta fuente, por lo que se hace relevante profundizar en estos estudios.

\section{REFERENCIAS}

Alcaldía Santiago de Cali (2015). Informe epidemiológico consolidado año 2015. Secretaría de Salud Pública. Informe. Santiago de Cali, Colombia, 74 pp.

Alcaldía Santiago de Cali (2016). Boletín epidemiológico No. 8 semana epidemiológica No. 30. Secretaría de Salud Municipal. Informe. Santiago de Cali, Colombia, 23 pp.

Bedner M. y MacCrehan W. A. (2006). Transformation of acetaminophen by chlorination produces the toxicants 1, 4-benzoquinone and $\mathrm{N}$-acetyl-p-benzoquinone imine. Environ. Sci. Technol. 40 (2), 516-522.

DOI: 10.1021/es0509073

Burks H., Pashos N., Martin E., Mclachlan J., Bunnell B. y Burow M. (2016). Endocrine disruptors and the tumor microenvironment: A new paradigm in breast cancer biology. Mol. Cell. Endocrinol. 1-7.

DOI: $10.1016 /$ j.mce.2016.12.010

Carballa M., Omil F. y Lema J. M. (2005). Removal of cosmetic ingredients and pharmaceuticals in sewage primary treatment. Water Res. 39 (19), 4790-4796. DOI: $10.1016 /$ j.watres.2005.09.018

Castro-Correia C. y Fontoura M. (2015). A influência da exposição ambiental a disruptores endócrinos no crescimento e desenvolvimento de crianças e adolescentes. Rev. Port. End. Diab. Metab. 10 (2), 186-192.

DOI: $10.1016 /$ j.rpedm.2014.10.002

Chen M., Ohman K., Metcalfe C., Ikonomou M. G., Amatya P. L. y Wilson J. (2006). Pharmaceuticals and endocrine disruptors in wastewater treatment effluents and in the water supply system of Calgary, Alberta, Canada. Water Qual. Res. J. Can. 41 (4), 351-364.

CONPES (2013). CONPES 3750 de 2013. Importancia estratégica del proyecto "apoyo financiero al plan de inversiones en infraestructura para fortalecer la prestación de los servicios de acueducto y 
alcantarillado en el municipio de Santiago de Cali". Consejo Nacional de Política Económica y Social. Bogotá, D.C., Colombia, 26 pp.

de Jesus Gaffney V., Almeida C. M., Rodrigues A., Ferreira E., Benoliel M. J. y Cardoso V. V. (2015). Occurrence of pharmaceuticals in a water supply system and related human health risk assessment. Water Res. 72, 199-208. DOI: $10.1016 /$ j.watres.2014.10.027

DNP (2016). Retos y perspectivas en las estructuración de proyectos y la gestión contractual de la inversión pública en Colombia. Departamento Nacional de Planeación. Bogotá, D.C., Colombia, 91 pp.

Durán-Alvarez J. C., Becerril-Bravo E., Castro V. S., Jiménez B. y Gibson R. (2009). The analysis of a group of acidic pharmaceuticals, carbamazepine, and potential endocrine disrupting compounds in wastewater irrigated soils by gas chromatography-mass spectrometry. Talanta 78 (3), 1159-1166. DOI: $10.1016 /$ j.talanta.2009.01.035

EPHC (2007). Australian guidelines for water recycling: managing health and environmental risks (Phase 2). Augmentation of drinking water supplies. Environment Protection and Heritage Council. Proyecto para comentarios del público [en línea]. https://www.nhmrc. gov.au/_files_nhmrc/publications/attachments/eh56_ water_recycling_guidelines_augmentation_drinking_supplies_22.pdf 19/09/2017.

Fenet H., Mathieu O., Mahjoub O., Li Z., Hillaire-Buys D., Casellas C. y Gomez E. (2012). Carbamazepine, carbamazepine epoxide and dihydroxycarbamazepine sorption to soil and occurrence in a wastewater reuse site in Tunisia. Chemosphere 88 (1), 49-54. DOI: 10.1016/j.chemosphere.2012.02.050

Flint S., Markle T., Thompson S. y Wallace E. (2012). Bisphenol A exposure, effects, and policy: a wildlife perspective. J. Environ. Manage. 104, 19-34.

DOI: 10.1016/j.jenvman.2012.03.021

Flippin J. L., Huggett D. y Foran C. M. (2007). Changes in the timing of reproduction following chronic exposure to ibuprofen in Japanese medaka, Oryzias latipes. Aquat. Toxicol. 81 (1), 73-78.

DOI: $10.1016 /$ j.aquatox.2006.11.002

Grenni P., Patrolecco L., Ademollo N., Tolomei A. y Caracciolo A. B. (2013). Degradation of gemfibrozil and naproxen in a river water ecosystem. Microchem. J. 107, 158-164. DOI: 10.1016/j.microc.2012.06.008

Han S., Choi K., Kim J., Ji K., Kim S., Ahn B., Yun J., Choi K., Khim J. S., Zhang X. y Giesy J.P. (2010). Endocrine disruption and consequences of chronic exposure to ibuprofen in Japanese medaka (Oryzias latipes) and freshwater cladocerans Daphnia magna and Moina macrocopa. Aquat. Toxicol. 98 (3), 256264. DOI: $10.1016 /$ j.aquatox.2010.02.013
Hernando M. D., Mezcua M., Fernández-Alba A. R. y Barceló D. (2006). Environmental risk assessment of pharmaceutical residues in wastewater effluents, surface waters and sediments. Talanta 69 (2), 334-342. DOI: $10.1016 /$ j.talanta.2005.09.037

Houeto P., Carton A., Guerbet M., Mauclaire A.C., Gatignol C., Lechat P. y Masset D. (2012). Assessment of the health risks related to the presence of drug residues in water for human consumption: Application to carbamazepine. Regul. Toxicol. Pharm. 62 (1), 41-48. DOI: 10.1016/j.yrtph.2011.11.012

INVIMA (2016). Listado de medicamentos de venta libre. Instituto Nacional de Vigilancia de Medicamentos y Alimentos [en línea]. https://www.datos.gov.co/Saludy-Protecci-n-Social/LISTADO-DE-MEDICAMENTOS-EN-VENTA-LIBRE/xzwX-qpja/data 04/09/2017.

Jürgens M.D., Holthaus K.I., Johnson A.C., Smith J.J., Hetheridge M. y Williams R.J. (2002). The Potential for Estradiol and Ethinylestradiol Degradation in English Rivers. Environ. Toxicol. Chem. 21 (3), 480-488. DOI: $10.1002 /$ etc. 5620210302

Kabir E. R., Rahman M. S. y Rahman I. (2015). A review on endocrine disruptors and their possible impacts on human health. Environ. Toxicol. Pharmacol. 40 (1), 241-258. DOI: 10.1016/j.etap.2015.06.009

Kim S. D., Cho J., Kim I. S., Vanderford B. J. y Snyder S. A. (2007). Occurrence and removal of pharmaceuticals and endocrine disruptors in South Korean surface, drinking, and waste waters. Water Res. 41 (5), 1013-1021.

DOI: 10.1016/j.watres.2006.06.034

Kleywegt S., Pileggi V., Yang P., Hao C., Zhao X., Rocks C., Thach S., Cheung P. y Whitehead B. (2011). Pharmaceuticals, hormones and bisphenol $\mathrm{A}$ in untreated source and finished drinking water in Ontario, Canada-occurrence and treatment efficiency. Sci. Total Environ. 409 (8), 1481-1488.

DOI: $10.1016 /$ j.scitotenv.2011.01.010

Koumaki E., Mamais D. y Noutsopoulos C. (2017). Environmental fate of non-steroidal anti-inflammatory drugs in river water/sediment systems. J. Hazard. Mater. 323, 233-241.

DOI: 10.1016/j.jhazmat.2016.03.026

Kumar A. y Xagoraraki I. (2010). Pharmaceuticals, personal care products and endocrine-disrupting chemicals in US surface and finished drinking waters: a proposed ranking system. Sci. Total Environ. 408 (23), 59725989. DOI: $10.1016 /$ j.scitotenv.2010.08.048

Locatelli M. A. F., Sodré F. F. y Jardim W. F. (2011). Determination of antibiotics in brazilian surface waters using liquid chromatography-electrospray tandem mass spectrometry. Arch. Environ. Contam. Toxicol. 60 (3), 385-393. DOI: 10.1007/s00244-010-9550-1 
López J. J., Dennis R. y Moscoso S. M. (2009). A study of self-medication in a neighborhood in Bogotá. Revista de Salud Pública 11, 432-442.

Miao X.S. y Metcalfe C. D. (2003). Determination of carbamazepine and its metabolites in aqueous samples using liquid chromatography-electrospray tandem mass spectrometry. Anal. Chem. 75 (15), 3731-3738. DOI: $10.1021 / \mathrm{ac} 030082 \mathrm{k}$

Michael I., Rizzo L., McArdell C. S., Manaia C. M., Merlin C., Schwartz T., Dagot C. y Fatta-Kassinos D. (2013). Urban wastewater treatment plants as hotspots for the release of antibiotics in the environment: a review. Water Res. 47 (3), 957-995.

DOI: $10.1016 /$ j.watres.2012.11.027

MINSALUD (2012). Resolución 4143 de 2012. Por la cual se establece el reglamento técnico sobre los requisitos sanitarios que deben cumplir los materiales, objetos, envases y equipamientos plásticos y elastoméricos y sus aditivos, destinados a entrar en contacto con alimentos y bebidas para consumo humano en el territorio nacional. Ministerio de Salud y Protección Social. Diario Oficial 48642. 12 de diciembre de 2012.

MINSALUD (2015). Resolución 5592 de 2015. Por la cual se actualiza integralmente el Plan de Beneficios en Salud con cargo a la Unidad de Pago por CapitaciónUPC del Sistema General de Seguridad Social en Salud - SGSSS y se dictan otras disposiciones. Ministerio de Salud y Protección Social. Diario Oficial 49739. 28 de diciembre de 2015.

Moermond C. (2014). Environmental risk limits for pharmaceuticals: Derivation of WFD water quality standards for carbamazepine, metoprolol, metformin and amidotrizoic acid. National Institute for Public Health and the Environment. Reporte. Rijksinstituut voor Volksgezondheid en Milieu letter report 270006002, Bilthoven, Holanda, 56 pp.

Mompelat S., Le Bot B. y Thomas O. (2009). Occurrence and fate of pharmaceutical products and by-products, from resource to drinking water. Environ Int. 35 (5), 803-814.

DOI: 10.1016/j.envint.2008.10.008

Montoya C., Loaiza D., Torres P., Cruz C. H. y Escobar J. C. (2013). Efecto del incremento en la turbiedad del agua cruda sobre la eficiencia de procesos convencionales de potabilización (effect of increase of raw water turbidity on efficiency of conventional drinking water treatment processes). Revista EIA Escuela de Ingeniería de Antioquia 16, 137-148.

Ömeroğlu S. y Sanin F. D. (2014). Fate and degradation kinetics of nonylphenol compounds in aerobic batch digesters. Water Res. 64, 1-12.

DOI: $10.1016 /$ j.watres.2014.06.033
Parent A.S., Franssen D., Fudvoye J., Gérard A. y Bourguignon J.P. (2015). Developmental variations in environmental influences including endocrine disruptors on pubertal timing and neuroendocrine control: revision of human observations and mechanistic insight from rodents. Front. Neuroendocrinol. 38, 12-36.

DOI: 10.1016/j.yfrne.2014.12.004

Parlamento Europeo y Consejo de la Unión Europea (2008). Directiva 2008/105/CE. Relativa a las normas de calidad ambiental en el ámbito de la política de aguas, por la que se modifican y derogan ulteriormente las Directivas 82/176/CEE, 83/513/CEE, 84/156/CEE, 84/491/CEE y 86/280/CEE del Consejo, y por la que se modifica la Directiva 2000/60/CE. Diario oficial de la Unión Europea. 16 de diciembre de 2008.

Peng Y.H., Chen Y.J., Chang Y.J. y Shih Y.h. (2015). Biodegradation of bisphenol A with diverse microorganisms from river sediment. J. Hazard. Mater. 286, 285-290.

DOI: 10.1016/j.jhazmat.2014.12.051

Pérez-Vidal A., Delgado-Cabrera L.G. y Torres-Lozada P. (2012). Evolución y perspectivas del sistema de abastecimiento de la ciudad de Santiago de Cali frente al aseguramiento de la calidad del agua potable. Ingeniería y competitividad 14 (2), 69-81.

Radke M., Lauwigi C., Heinkele G., Mürdter T. E. y Letzel M. (2009). Fate of the antibiotic sulfamethoxazole and its two major human metabolites in a water sediment test. Environ. Sci. Technol. 43 (9), 3135-3141. DOI: 10.1021/es900300u

Rocco L., Frenzilli G., Zito G., Archimandritis A., Peluso C. y Stingo V. (2012). Genotoxic effects in fish induced by pharmacological agents present in the sewage of some Italian water-treatment plants. Environ Toxicol. 27(1), 18-25. DOI: 10.1002/tox.20607

Schwab B. W., Hayes E. P., Fiori J. M., Mastrocco F. J., Roden N. M., Cragin D., Meyerhoff R. D., D'Aco V.J. y Anderson P. D. (2005). Human pharmaceuticals in US surface waters: a human health risk assessment. Regul Toxicol Pharmacol. 42 (3), 296-312.

DOI: 10.1016/j.yrtph.2005.05.005

Simazaki D., Kubota R., Suzuki T., Akiba M., Nishimura T. y Kunikane S. (2015). Occurrence of selected pharmaceuticals at drinking water purification plants in Japan and implications for human health. Water Res. 76, 187-200.

DOI: 10.1016/j.watres.2015.02.059

Snyder S. A., Adham S., Redding A. M., Cannon F. S., DeCarolis J., Oppenheimer J., Wert E. C. y Yoon Y. (2007). Role of membranes and activated carbon in the removal of endocrine disruptors and pharmaceuticals, Desalination 202 (1-3), 156-181.

DOI: $10.1016 /$ j.desal.2005.12.052 
Soufan M., Deborde M., Delmont A. y Legube B. (2013). Aqueous chlorination of carbamazepine: kinetic study and transformation product identification. Water Res. 47 (14), 5076-5087.

DOI: 10.1016/j.watres.2013.05.047

Su T., Deng H., Benskin J. P. y Radke M. (2016). Biodegradation of sulfamethoxazole photo-transformation products in a water/sediment test. Chemosphere 148, 518-525. DOI: 10.1016/j.chemosphere.2016.01.049

Sweeney M. F., Hasan N., Soto A. M. y Sonnenschein C. (2015). Environmental endocrine disruptors: effects on the human male reproductive system. Rev. Endocr. Metab. Disord. 16 (4), 341-357.

DOI: $10.1007 / \mathrm{s} 11154-016-9337-4$

Toro-Vélez A. F., Madera-Parra C. A., Peña-Varón M. R., Lee W. Y., Bezares-Cruz J. C., Walker W. S., CárdenasHenao H., Quesada-Calderón S., García-Hernández H. y Lens P. N. L. (2016). BPA and NP removal from municipal wastewater by tropical horizontal subsurface constructed wetlands. Sci. Total Environ. 542, 93-101. DOI: $10.1016 /$ j.scitotenv.2015.09.154

USEPA (2016). Contaminant candidate list (CCL) and regulatory determination. United States Environmental Protection Agency [en línea]. https://www.epa.gov/ccl/ chemical-contaminants-ccl-4 07/01/2017

Vanderford B. J., Pearson R. A., Rexing D. J. y Snyder S. A. (2003). Analysis of endocrine disruptors, pharmaceuticals, and personal care products in water using liquid chromatography/tandem mass spectrometry. Anal. Chem. 75 (22), 6265-6274.

DOI: $10.1021 / \mathrm{ac} 034210 \mathrm{~g}$

Virkutyte J., Varma R. S. y Jegatheesan V. (2010). Treatment of micropollutants in water and wastewater. IWA Publishing, Londres, Reino Unido, 483 pp.

Wenzel A., Böhmer W., Müller J., Rüdel H. y SchrÖterKermani C. (2004). Retrospective monitoring of alkylphenols and alkylphenol monoethoxylates in aquatic biota from 1985 to 2001: results from the German Environmental Specimen Bank. Environ. Sci. Technol. 38 (6), 1654-1661.

DOI: $10.1021 / \mathrm{es} 035032 \mathrm{~b}$
Westerhoff P., Yoon Y., Snyder S. y Wert E. (2005). Fate of endocrine-disruptor, pharmaceutical, and personal care product chemicals during simulated drinking water treatment processes. Environ. Sci. Technol. 39 (17), 6649-6663. DOI: 10.1021/es0484799

Wielogórska E., Elliott C. T., Danaher M., Chevallier O. y Connolly L. (2015). Validation of an ultra high performance liquid chromatography-tandem mass spectrometry method for detection and quantitation of 19 endocrine disruptors in milk. Food Control 48, 48-55. DOI: 10.1016/j.foodcont.2014.06.001

Wiklund O., Angelin B., Bergman M., Berglund L., Bondjers G., Carlsson A., Linden T., Miettinen T., Ödman B., Olofsson S.O., Saarinen I., Sipilä R., Sjöström P., Kron B., Vanhanen H. y Wrigth I. (1993). Pravastatin and gemfibrozil alone and in combination for the treatment of hypercholesterolemia. Am. J. Med. 94 (1), 13-20. DOI: 10.1016/0002-9343(93)90114-5

Xu B., Mao D., Luo Y. y Xu L. (2011). Sulfamethoxazole biodegradation and biotransformation in the watersediment system of a natural river. Bioresour. Technol. 102 (14), 7069-7076.

DOI: 10.1016/j.biortech.2011.04.086

Xu J., Wang P., Guo W., Dong J., Wang L. y Dai S. (2006). Seasonal and spatial distribution of nonylphenol in Lanzhou Reach of Yellow River in China. Chemosphere 65 (9), 1445-1451.

DOI: $10.1016 /$ j.chemosphere.2006.04.042

Ying G.G., Kookana R. S. y Ru Y.J. (2002). Occurrence and fate of hormone steroids in the environment. Environ. int. 28 (6), 545-551. DOI: $10.1016 / \mathrm{S} 0160-4120(02) 00075-2$

Zhang Y., Geißen S.U. y Gal C. (2008). Carbamazepine and diclofenac: removal in wastewater treatment plants and occurrence in water bodies. Chemosphere 73 (8), 1151-1161. DOI: 10.1016/j.chemosphere.2008.07.086

Zhou H., Zhou Y., Li H. y Wang F. (2012). Fate and removal of selected endocrine-disrupting compounds in sewage using activated sludge treatment. Water Environ. J. 26 (4), 435-444.

DOI: $10.1111 / \mathrm{j} .1747-6593.2011 .00302 . x$ 